\title{
Dermatitis granulomatosa neutrofílica en empalizada como presentación inicial de lupus eritematoso sistémico
}

\author{
Katia Henostroza-Inga ${ }^{1 *}$, Rosario Torres-Ibérico ${ }^{1}$, Noé Atamari-Anahui ${ }^{1,2}$ y Roxana Lipa-Chancolla ${ }^{3}$ \\ ${ }^{1}$ Servicio de Dermatología, Instituto Nacional de Salud del Niño-Breña; ${ }^{2}$ Unidad de Investigación para la Generación y Síntesis de Evidencias en \\ Salud, Vicerrectorado de Investigación, Universidad San Ignacio de Loyola; ${ }^{3}$ Servicio de Anatomía Patológica, Instituto Nacional de Salud del \\ Niño-San Borja. Lima, Perú
}

\begin{abstract}
Resumen
Introducción: La dermatitis granulomatosa neutrofilica en empalizada es una manifestación cutánea poco frecuente en la infancia que se asocia con patologías autoinmunitarias. La patogénesis exacta de esta enfermedad aún se desconoce. Sin embargo, se ha sugerido que el depósito de complejos inmunitarios podría iniciarla. Caso clínico: Se describe el caso de una paciente de 11 años que presentó lesiones polimórficas en las extremidades asociadas a poliartralgias. Fue diagnosticada de lupus eritematoso sistémico y nefritis lúpica. Por las lesiones en la piel, se realizaron estudios histopatológico e inmunohistoquímico (CD68, CD163, mieloperoxidasa), que dieron como resultado dermatitis granulomatosa neutrofilica en empalizada. Debido al compromiso renal, se administró tratamiento con pulsos de metilprednisolona, además de hidroxicloroquina, micofenolato de mofetilo, antihipertensivos y antiinflamatorios no esteroideos. La respuesta clínica fue favorable durante el seguimiento. Conclusiones: La dermatitis granulomatosa neutrofilica en empalizada asociada a lupus eritematoso sistémico es inusual. Por ello, es importante su reconocimiento, ya que puede presentarse como manifestación inicial de la enfermedad autoinmunitaria.
\end{abstract}

Palabras clave: Dermatitis granulomatosa neutrofilica en empalizada. Dermatitis granulomatosa intersticial. Lupus eritematoso sistémico. Niños.

\section{Palisaded neutrophilic granulomatous dermatitis as the initial presentation of systemic lupus erythematosus}

\begin{abstract}
Background: Palisaded neutrophilic granulomatous dermatitis is a rare cutaneous manifestation in children associated with autoimmune pathologies. The exact pathogenesis of this disease is still unknown. However, it has been suggested that the deposition of immune complexes could initiate this pathology. Case report: We describe the case of an 11-yearold female patient who presented with polymorphic lesions in extremities associated with polyarthralgia. She was diagnosed with systemic lupus erythematosus and lupus nephritis. Because of the skin lesions, histopathological and immunohistochemical skin studies (CD68, CD163, myeloperoxidase) were performed, which resulted in palisaded neutrophilic granulomatous dermatitis. Due to renal involvement, treatment was administered with methylprednisolone pulses, hydroxychloroquine, mycophenolate mofetil, antihypertensives, and nonsteroidal anti-inflammatory drugs. The clinical response was favorable during follow-up. Conclusions: Palisaded neutrophilic granulomatous dermatitis associated with systemic
\end{abstract}

Correspondencia:

*Katia Henostroza-Inga

E-mail: katicita_85@ hotmail.com
Fecha de recepción: 19-02-2021

Fecha de aceptación: 03-05-2021

DOI: 10.24875/BMHIM.21000035
Disponible en internet: 16-12-2021

Bol Med Hosp Infant Mex. 2021;78(6):652-656

www.bmhim.com

1665-1146/C 2021 Hospital Infantil de México Federico Gómez. Publicado por Permanyer. Este es un artículo open access bajo la licencia CC BY-NC-ND (http://creativecommons.org/licenses/by-nc-nd/4.0/). 
lupus erythematosus is unusual. Therefore, its recognition is important, as it may appear as the initial manifestation of this autoimmune disease.

Keywords: Palisaded neutrophilic granulomatous dermatitis. Interstitial granulomatous dermatitis. Systemic lupus erythematosus. Child.

\section{Introducción}

La dermatitis granulomatosa neutrofílica en empalizada (DGNP) es una rara erupción cutánea polimórfica y simétrica'. En 1951 fue descrita como granuloma de Churg-Strauss, y en 1996 Wilmoth y Perniciaro confirmaron su asociación con algunas enfermedades sistémicas en la población adulta, como artritis reumatoide y poliangeítis granulomatosa, entre otras ${ }^{2}$. Aunque los reportes de DGNP en pacientes pediátricos son raros, algunos han descrito su asociación con enfermedades sistémicas autoinmunitarias, como esclerosis sistémica y granulomatosis eosinofílica con poliangeítis ${ }^{3}$.

La asociación entre DGNP y lupus eritematoso sistémico (LES) ha sido poco descrita. Terai et al. ${ }^{4}$ reportaron una mayor frecuencia de esta asociación en adultos jóvenes (media de edad de: 36 años), con predominio del sexo femenino, acompañada de nefritis lúpica en la mayoría de los casos, mientras que Larson y Granter ${ }^{5}$ estudiaron 14 pacientes (media de edad de: 41 años; 13 mujeres) con LES y dermatosis neutrofílica y solo encontraron un paciente con características de DGNP.

Debido a la diversidad de manifestaciones cutáneas, el diagnóstico resulta complicado, por lo que se requiere realizar un estudio histopatológico.

El objetivo de este artículo es reportar un caso de DGNP como manifestación inicial en una paciente de 11 años con debut de LES.

\section{Caso clínico}

Paciente de 11 años, de sexo femenino, que 4 meses antes de su ingreso a la institución presentó poliartralgias e hiporexia, 2 meses después aparecieron lesiones polimórficas en las zonas extensoras de los miembros superiores e inferiores, y 1 mes antes de su hospitalización presentó diariamente fiebre y debilidad generalizada que le dificultaba la deambulación.

Entre los antecedentes familiares se informaron psoriasis y fibromialgias. El esquema de vacunación estaba completo. No se reportaron hospitalizaciones previas, enfermedades ni intervenciones quirúrgicas.

En la exploración física se encontró: frecuencia cardiaca 105 latidos/min, frecuencia respiratoria 22

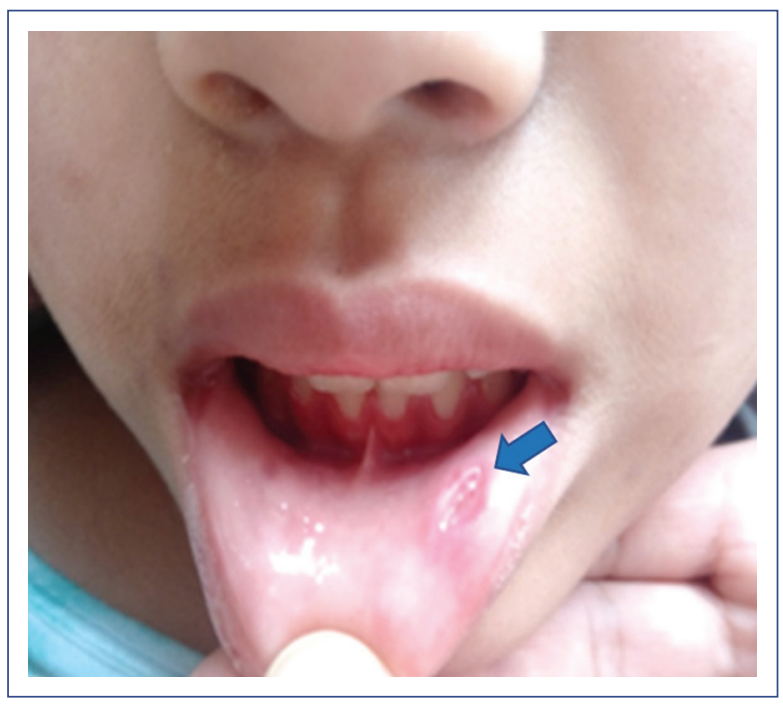

Figura 1. Úlcera de base eritematosa y bordes definidos en la mucosa yugal inferior (flecha).

respiraciones/min, presión arterial 105/80 mmHg, temperatura $37.2^{\circ} \mathrm{C}$, peso $48 \mathrm{~kg}$ y talla $147 \mathrm{~cm}$. Se observó palidez en piel y mucosas $(++/+++)$, una úlcera no sangrante en la mucosa oral (Figura 1) y lesiones pápulo-eritematosas y vesículo-costrosas violáceas distribuidas de manera simétrica y bilateral en la zona extensora de los miembros superiores e inferiores (Figuras 2 y 3). En el examen neurológico, la puntuación en la escala de Glasgow fue 15/15, la fuerza muscular $4 / 5$ en todas las extremidades y el tono muscular conservado, con reflejos rotulianos presentes. El resto del examen, sin alteraciones.

En las pruebas de laboratorio se encontró: hemoglobina $8.6 \mathrm{~g} / \mathrm{dL}$, leucocitos $5170 / \mathrm{mm}^{3}$, linfocitos 775 (valores normales [VN]:1500-6500), plaquetas $227,000 / \mathrm{mm}^{3}$, proteína $C$ reactiva $5.65 \mathrm{mg} / \mathrm{dL}$ y velocidad de sedimentación globular $60 \mathrm{~mm} / \mathrm{h}$. La prueba de Coombs directa fue positiva, y además se encontraron $291 \mathrm{mg} /$ $\mathrm{dL}$ de haptoglobina (VN: 30-200), $806 \mathrm{U} / \mathrm{L}$ de lactato deshidrogenasa (VN: 230-460), $21 \mathrm{mg} / \mathrm{dL}$ de complemento C3 (VN: 90-180), 2 mg/dL de C4 (VN: 10-40), $7309 \mathrm{mg} / \mathrm{mL}$ de B2 microglobulina (VN: 699-1836), $54 \mathrm{mg} / \mathrm{dl}$ de urea (VN:10-38), $1.03 \mathrm{mg} / \mathrm{dL}$ de creatinina (VN: 0.3-0.7) y proteinuria de $2315 \mathrm{mg} / 24 \mathrm{~h}$ 


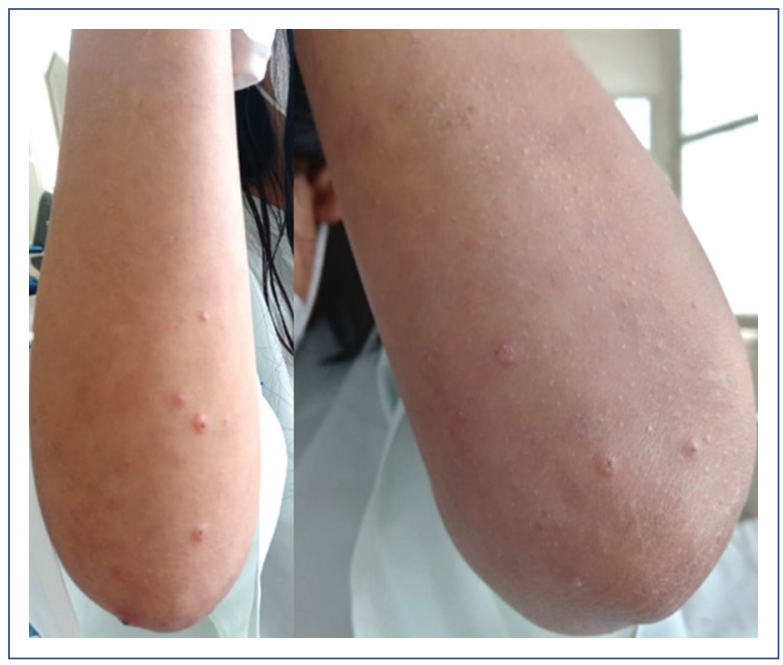

Figura 2. Pápulas con costras centrales, simétricas, en la superficie extensora de los miembros superiores.

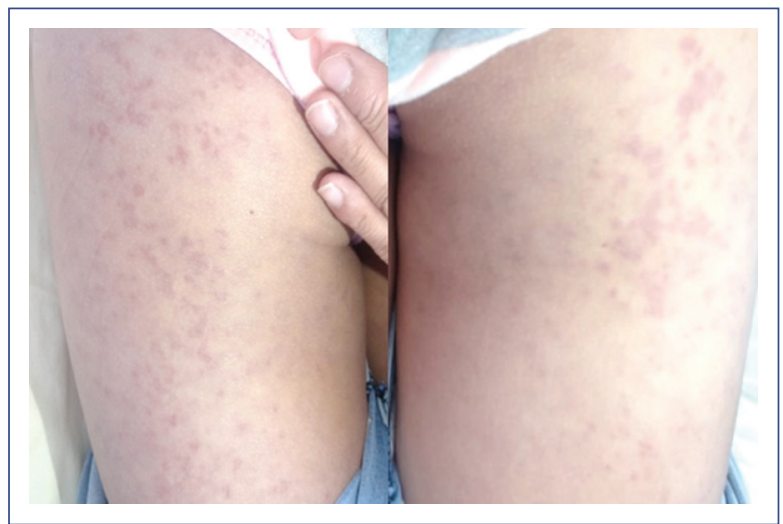

Figura 3. Placas eritemato-violáceas simétricas en la región posterior de los muslos.

$\left(65.6 \mathrm{mg} / \mathrm{m}^{2} / \mathrm{h}\right)$. Los electrolitos séricos estaban dentro de los valores normales y en el examen de orina se encontró hematuria. Las pruebas inmunológicas fueron positivas para anticuerpos antinucleares (título de 1:100), con patrón homogéneo y citoplasmático. El perfil de antígeno nuclear extraíble fue positivo para anticuerpos dsDNA, RNP/Sm, Sm, nucleosomas, histonas y proteína P-ribosomal.

Con respecto a las lesiones en la piel, en el estudio histopatológico se encontró una dermis reticular con abundantes neutrófilos, polvo nuclear y degeneración basófila del colágeno, vasos con edema intenso de células endoteliales con fibrina de la pared, ambos rodeados por histiocitos e infiltrado linfomononuclear leve que se extiende y sigue a los anexos (Figura 4).

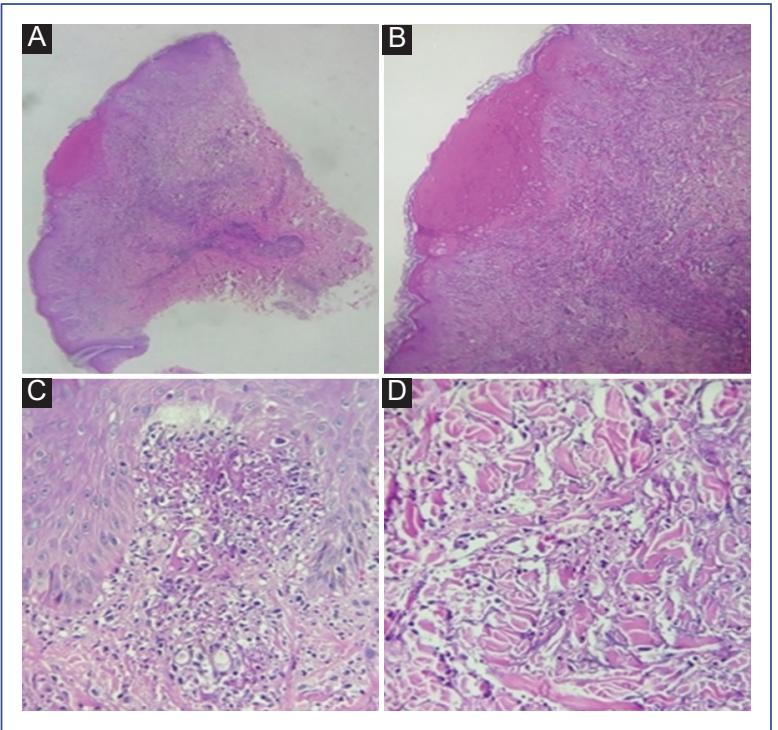

Figura 4. Imágenes macroscópica (A) y microscópica (B) en las que se observa un infiltrado linfomononuclear en la dermis y los anexos (tinción de hematoxilina-eosina, $\times 100$ ). C) En la dermis reticular se observan abundantes neutrófilos y polvo nuclear (tinción de hematoxilinaeosina, $\times 200)$. D) Degeneración basófila de colágeno y vasos con edema intenso de células endoteliales con fibrina de la pared (tinción de hematoxilina-eosina, $\times 400$ ).

El estudio mediante tinción HQ Alcian Blue fue positivo, mientras que la inmunohistoquímica detectó CD68 y CD163 positivos en histiocitos y mieloperoxidasa positiva en el infiltrado neutrofílico (Figura 5).

También se realizaron otros exámenes complementarios, como radiografía de tórax, prueba de tuberculina, baciloscopia directa en esputo y tinción con ácido peryódico de Schiff en la biopsia de piel, para descartar Mycobacterium tuberculosis y hongos como causa de los granulomas, los cuales fueron negativos.

Con los hallazgos clínicos, histopatológicos y de laboratorio, la paciente fue diagnosticada de DGNP asociada a debut de LES con afectación renal. Se realizó una biopsia renal que reveló nefritis lúpica membranoproliferativa difusa de clase IV A/C, puntaje de actividad 9 y cronicidad 2.

Como tratamiento, se le administraron pulsos de metilprednisolona (30 mg/kg/día por 3 días), para continuar con prednisona (1 mg/kg/día), micofenolato de mofetilo (1200 mg/m²/día), hidroxicloroquina (4 mg/kg/día) y enalapril $(0.5 \mathrm{mg} / \mathrm{kg} / \mathrm{día})$ de manera permanente, y se agregaron paracetamol $(15 \mathrm{mg} / \mathrm{kg} /$ dosis) o naproxeno (10 mg/kg/dosis) según el dolor. La paciente respondió adecuadamente: las lesiones 


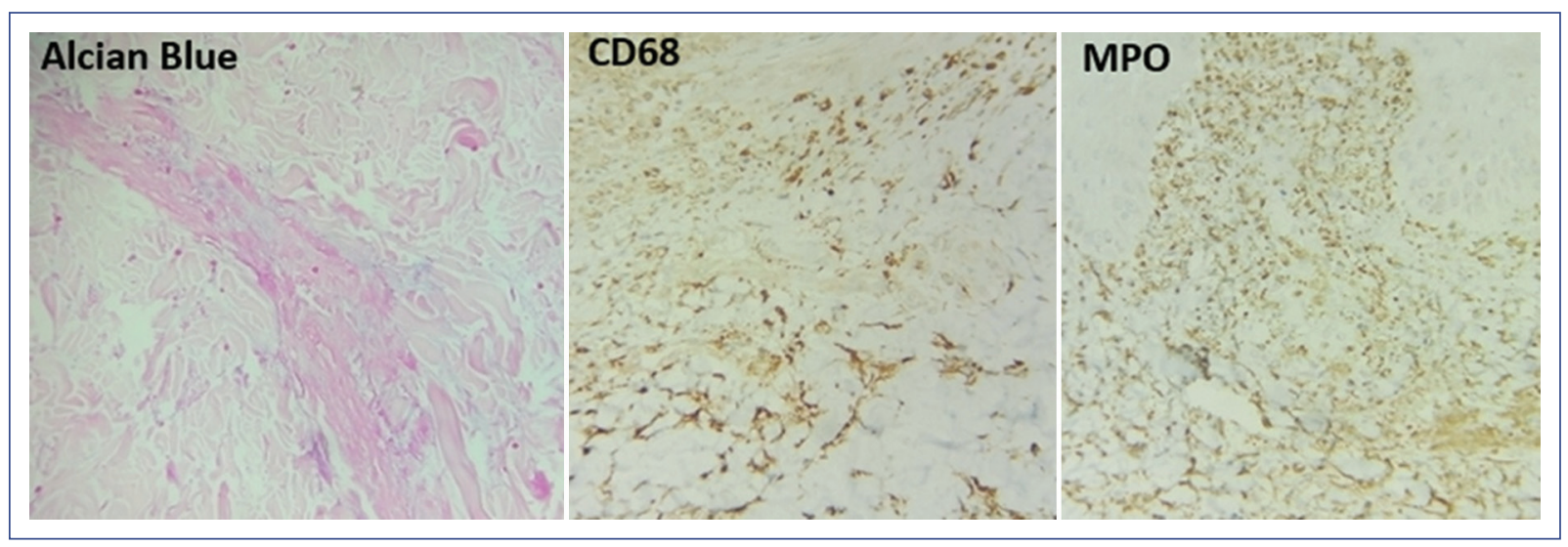

Figura 5. Estudio inmunohistoquímico: tinción HO Alcian Blue positiva, marcador CD68 positivo en histiocitos y mieloperoxidasa positiva en infiltrado neutrofílico.

desaparecieron y se observó una mejoría de la función renal a las 2 semanas de iniciado el tratamiento. En el seguimiento, la paciente continúa sus controles por reumatología, nefrología y dermatología, con la misma medicación, sin presentar actividad del LES ni aparición de nuevas lesiones en la piel.

\section{Discusión}

Las dermatosis neutrofílicas son trastornos poco frecuentes en la infancia, que se caracterizan por la presencia de infiltrados neutrofílicos estériles en la piel y comparten aspectos clínicos similares ${ }^{6-8}$. El término general incluye dermatitis granulomatosa intersticial, granuloma de Churg-Strauss y reacción intersticial granulomatosa por fármacos ${ }^{9}$.

Aunque la patogenia exacta de esta enfermedad aún se desconoce, se ha sugerido que, debido a una patología autoinmunitaria de base, ciertos inmunocomplejos se depositan en los vasos dérmicos, desencadenando una serie de eventos como liberación de quimiocinas y de factor de necrosis tumoral. A su vez, esto conllevaría procesos de inflamación, daño del colágeno e infiltrado granulomatoso en la dermis intersticial y perivascular ${ }^{10}$. Por lo tanto, el proceso comienza como una vasculitis leucocitoclástica que luego progresa a la degeneración del colágeno seguida de inflamación crónica y fibrosis. Todo esto causaría el espectro de características clínicas e histológicas. También se mencionan, como causas secundarias, fármacos como el adalimumab, inhibidores de la enzima convertidora de angiotensina, diuréticos, inhibidores del factor de necrosis tumoral y productos de soya ${ }^{11,12}$. En el presente caso, se puede suponer que la patología cutánea fue el resultado de un proceso inflamatorio que produjo el LES en los vasos sanguíneos de la dermis intersticial.

Dentro de los casos reportados de DGNP en adultos, se ha descrito la asociación con artritis reumatoidea en el $26.8 \%$ de los casos y con LES en el $11.3 \%{ }^{13}$. Esta asociación puede estar mediada por inmunocomplejos y correlacionada con el empeoramiento de la enfermedad subyacente. Además, se han informado casos de DGNP como forma de presentación de debut o brote de $\mathrm{LES}^{3}$, como ocurrió en esta paciente.

Clínicamente, la DGNP se caracteriza por lesiones polimórficas y heterogéneas, como máculas, placas (eritematosas de configuración anular) y nódulos de distribución simétrica en el tronco, las extremidades, los codos y la región proximal e interna de los brazos. Dentro de los síntomas asociados se describen artralgias y prurito, y en general el tiempo entre el diagnóstico de la patología de base y la presentación de las lesiones es menor de 1 año ${ }^{10}$.

En la histopatología se describe un infiltrado intersticial inflamatorio difuso, perivascular, superficial y profundo, compuesto principalmente por neutrófilos e histiocitos CD68 positivos ${ }^{10}$. También puede observarse una zona central de colágeno degenerado, rodeada por histiocitos y linfocitos en empalizada (asemeja a un granuloma anular), además de eosinófilos, células plasmáticas o células gigantes multinucleadas. La histoquímica puede revelar la presencia de Alcian Blue de forma escasa ${ }^{10}$. En la paciente estudiada se observaron características similares a las descritas en la literatura, como el infiltrado inflamatorio compuesto por gran cantidad de neutrófilos, vasos rodeados por histiocitos y linfocitos con Alcian Blue positivo, que demostraría el depósito de mucina intersticial 
observado en los pacientes con LES, además de CD68 positivo en histiocitos (por inmunohistoquímica).

Es importante distinguir las lesiones características de DGNP de otras afecciones con una apariencia clínica o histológica similar. Por ello, dentro de los diagnósticos diferenciales hay que considerar el lupus eritematoso túmido, el eritema nudoso, el síndrome de Sweet y el granuloma anular ${ }^{14}$.

El tratamiento se basa en controlar la enfermedad subyacente y esteroides tópicos y sistémicos, inmunosupresores como la dapsona, la ciclofosfamida, el micofenolato de mofetilo, la ciclosporina, el metotrexato, la colchicina, la hidroxicloroquina y el infliximab, y antiinflamatorios no esteroideos (AINE). La respuesta al tratamiento es variable, pero en la mayoría de los casos es satisfactoria ${ }^{15}$. La paciente recibió tratamiento con corticoides sistémicos, AINE, micofenolato de mofetilo e hidroxicloroquina, con completa involución de las lesiones de la piel y control de la nefropatía lúpica. Con respecto al compromiso renal, Terai et al. ${ }^{4}$ encontraron que la presencia de nefritis lúpica de clase II y V fue una característica frecuente en los pacientes con DGNP, como en nuestra paciente (clase IV). Este hallazgo demostraría una relación entre las lesiones en la piel y el compromiso renal en pacientes con debut de LES.

El presente caso se considera importante porque apoya la hipótesis de que las lesiones de la piel en la DGNP podrían adelantar el compromiso renal en el LES, por lo que son un probable e importante factor pronóstico. Sin embargo, debido a la escasa casuística, son necesarios estudios con mejor metodología para fortalecer esta posibilidad.

En conclusión, la DGNP es una manifestación cutánea asociada con patologías autoinmunitarias poco frecuente en la infancia. Es importante su reconocimiento, ya que puede presentarse como una manifestación inicial de LES. Aunque la DGNP en pacientes con LES se relaciona de manera incierta con la evolución de la enfermedad, la presencia de nefritis lúpica se ha descrito en reportes previos, lo que evidencia una relación que se debe considerar para futuros estudios.

\section{Responsabilidades éticas}

Protección de personas y animales. Los autores declaran que para esta investigación no se han realizado experimentos en seres humanos ni en animales.
Confidencialidad de los datos. Los autores declaran que han seguido los protocolos de su centro de trabajo sobre la publicación de datos de pacientes.

Derecho a la privacidad y consentimiento informado. Los autores han obtenido el consentimiento informado de los pacientes y sujetos referidos en el artículo. Este documento obra en poder del autor de correspondencia.

\section{Conflicto de intereses}

Los autores declaran no tener ningún conflicto de intereses.

\section{Financiamiento}

La presente investigación no ha recibido ayudas específicas provenientes de agencias del sector público, sector comercial ni entidades sin ánimo de lucro.

\section{Bibliografía}

1. Germanas JP, Mehrabi D, Carder KR. Palisaded neutrophilic granulomatous dermatitis in a 12-year-old girl with systemic lupus erythematosus. J Am Acad Dermatol. 2006;55(2 Suppl):S60-2.

2. Wilmoth GJ, Perniciaro C. Cutaneous extravascular necrotizing granuloma (Winkelmann granuloma): confirmation of the association with systemic disease. J Am Acad Dermatol. 1996;34(5 Pt 1):753-9.

3. Berk DR, Bayliss SJ. Neutrophilic dermatoses in children. Pediatr Dermatol. 2008;25:509-19.

4. Terai S, Ueda-Hayakawa I, Nguyen CTH, Ly NTM, Yamazaki F, Kambe N, et al. Palisaded neutrophilic and granulomatous dermatitis associated with systemic lupus erythematosus: possible involvement of CD163+ M2 macrophages in two cases, and a review of published works. Lupus. 2018;27:2220-7

5. Larson AR, Granter SR. Systemic lupus erythematosus-associated neutrophilic dermatosis - an underrecognized neutrophilic dermatosis in patients with systemic lupus erythematosus. Hum Pathol. 2014;45:598-605.

6. Saavedra AP, Kovacs SC, Moschella SL. Neutrophilic dermatoses. Clin Dermatol. 2006;24:470-81.

7. Gilliam AE. Skin signs of systemic disease in childhood. Adv Dermatol. 2006;22:1-30.

8. Nischal KC, Khopkar U. An approach to the diagnosis of neutrophilic dermatoses: a histopathological perspective. Indian J Dermatol Venereol Leprol. 2007;73:222-30.

9. Rosenbach M, English JC $3^{\text {rd }}$. Reactive granulomatous dermatitis: a review of palisaded neutrophilic and granulomatous dermatitis, interstitial granulomatous dermatitis, interstitial granulomatous drug reaction, and a proposed reclassification. Dermatol Clin. 2015;33:373-87.

10. Rodríguez-Garijo N, Bielsa I, Mascaró JM Jr, Quer A, Idoate MA, Paricio JJ, et al. Reactive granulomatous dermatitis as a histological pattern including manifestations of interstitial granulomatous dermatitis and palisaded neutrophilic and granulomatous dermatitis: a study of 52 patients. J Eur Acad Dermatol Venereol. 2021;35:988-94.

11. Collaris EJ, van Marion AM, Frank J, Poblete-Gutiérrez P. Cutaneous granulomas in rheumatoid arthritis. Int J Dermatol. 2007;46(Suppl 3):33-5.

12. Stephenson SR, Campbell SM, Drew GS, Magro CM. Palisaded neutrophilic and granulomatous dermatitis presenting in a patient with rheumatoid arthritis on adalimumab. J Cutan Pathol. 2011;38:644-8.

13. Hantash BM, Chiang D, Kohler S, Fiorentino D. Palisaded neutrophilic and granulomatous dermatitis associated with limited systemic sclerosis. J Am Acad Dermatol. 2008;58:661-4.

14. Maxfield LJ, Tanner LS, Schwartz C. Extensive palisaded neutrophilic granulomatous dermatitis with systemic lupus erythematosus. Skin J Cutan Med. 2020;4:260-4.

15. Maurelli M, Colato C, Girolomoni G. Palisaded neutrophilic granulomatous dermatitis and its associations with autoimmune diseases. Eur $\mathrm{J}$ Dermatol. 2019;29:432-3. 